Revista de Matemática: Teoría y Aplicaciones 2011 18(1) : 1-7

CIMPA - UCR ISSN: 1409-2433

\author{
INTEGRAL EQUATIONS FOR THE AGGREGATE \\ CLAIM AMOUNT
}

\title{
ECUACIONES INTEGRALES PARA EL MONTO AGREGADO DE RECLAMACIONES
}

\author{
Carlos G. Pacheco-González*
}

Received: 18 Feb 2010; Revised: 26 Aug 2010; Accepted: 10 Sep 2010

\begin{abstract}
In the context of insurance mathematics, we study the renewal properties of the so-called aggregate claim amount for the non-discounted and the discounted case. For these models, we set integral equations for the distribution function. Additionally we mention how the integral equation may be used to find an approximation of the distribution.
\end{abstract}

Keywords: aggregate claim amount, Volterra integral equation, discounted process.

\section{Resumen}

En el ámbito de matemáticas actuariales, estudiamos las propiedades de renovación del llamado monto agregado de reclamaciones en los casos no-descontado y descontado. Se establecen ecuaciones integrales

*Departamento de Matemáticas, CINVESTAV-IPN, A. Postal 14-740, México D.F. 07000, México. E-Mail: cpacheco@math.cinvestav.mx 
para la función de distribución de estos modelos. Adicionalmente mencionamos como usar estas ecuaciones integrales para encontrar aproximaciones numéricas de la distribución.

Palabras clave: monto agregado de reclamaciones, ecuación integral de Volterra, proceso de descuento.

Mathematics Subject Classification: 65C20, 65R20, 65D30.

\section{Introduction}

The classical model for the aggregate claim amount in an insurance company is given by the following random sum

$$
Z=\left\{Z_{t}=\sum_{i=1}^{N_{t}} X_{i}, t \geq 0\right\},
$$

where $X_{1}, X_{2}, \ldots$ is a sequence of independent identically distributed (i.i.d.) positive random variables (r.v.s), which represent the amount of the claim, and $N=\left\{N_{t}, t \geq 0\right\}$ is a renewal process, i.e.

$$
N_{t}=\max \left\{k: \sum_{i=1}^{k} \tau_{i} \leq t\right\},
$$

with $\tau_{1}, \tau_{2}, \ldots$ a sequence of i.i.d. positive r.v.s, usually called "interarrival times" (they represent the time between claims). We have the convention that $Z_{s}=0$ for $s<\tau_{1}$, i.e. before the first jump the process is 0 .

Using probabilistic arguments, one can easily find an integral equation for the distribution of $Z_{t}$. This integral equation classifies as a renewal type equation, amply discussed and use in applied probability. Also, there are numerous papers dealing with the numerical treatment of this equation.

Another popular model similar to (1) is the discounted aggregate claim amount, which takes into account the monetary present value of the cash flow. The model is given as

$$
Z^{(\delta)}=\left\{Z_{t}^{(\delta)}=\sum_{i=1}^{N_{t}} X_{i} e^{-\delta T_{i}}, t \geq 0\right\},
$$

where $N_{t}$ is defined as in (2), $T_{i}=\sum_{j=1}^{i} \tau_{j}$ (arrival times), $X_{1}, X_{2}, \ldots$ are i.i.d. positive r.v.s., and $\delta>0$ is the continuous time interest rate. In this paper we find an integral equation of Volterra type for $Z_{t}^{(\delta)}$.

Model (1) is well known, and it comes across in a great variety of applications; some references for this model are $[1,6]$. This random sum 
belongs to a general class of random summations known as continuoustime random walks, CTRWs for short (see for instance [7, 10]). Notice that process $Z$ is also called renewal-reward process (see [10]). There is a vast literature for this kind of processes, and when the interarrival times are exponentially distributed, process $Z$ is simply a compound Poisson process. By the renewal properties of the process, it is possible to derive an integral equation for the density of CTRWs, which is termed the master equation in Scalas et al [8]. Such equation is in general of Volterra type, and thus it becomes possible to solve it numerically by standard procedures. There are available in the literature methods for solving Volterra integral equations in two dimensions (see for example [3]). Othe related work is [9], where it is study the so-called ruin probabilities by means of a Thorin integral equation.

\section{The non-discounted sum}

We are interested in finding the distribution of $Z_{t}$, model (1):

$$
F(t, z):=P\left(Z_{t} \leq z\right) .
$$

Denote by $f_{X}(x)$ and $f_{\tau}(t)$ the density functions of $X_{1}$ and $\tau_{1}$, respectively. We also denote by $f_{W}(w \mid A)$ the density of the r.v. $W$ conditioned to the event $A$.

The following explanation is instructive for deriving integral equations for the density of $Z_{t}$, and also for $Z_{t}^{(\delta)}$.

Given the quantity $\tau_{1}+\ldots+\tau_{n}=T$ for any fixed $n \geq 1$, then

$$
N_{T+t}-n \stackrel{d}{=} N_{t}, t \geq 0 .
$$

This means that the process is renewed at the very moment of a jump. In general, the process $Z$ is not a Markov process and it does not have independent increments, but by assuming that $X_{1}, X_{2}, \ldots$ are i.i.d. and that $\sum_{i=1}^{n} X_{i}=z$ is given, it holds that

$$
Z_{T+t}-z \stackrel{d}{=} Z_{t}, t \geq 0
$$

Here $\stackrel{d}{=}$ stands for equality in distribution.

Proposition 1 Function $F$ satisfies the following Volterra linear integral equation in two dimensions:

$$
F(t, z)=P\left(\tau_{1}>t\right)+\int_{0}^{t} \int_{0}^{z} F(u, w) f_{X}(z-w) f_{\tau}(t-u) d w d u,
$$

for $t \geq 0$ and $z \geq 0$ 
Proof. The proof relies in the idea of splitting into independent events. In this case it is convenient to think of two cases: when the first jump occurs after time $t$, and when it occurs before. Thus, we have that

$$
F(t, z)=P\left(Z_{t} \leq z \mid \tau_{1}>t\right) P\left(\tau_{1}>t\right)+P\left(Z_{t} \leq z \mid \tau_{1} \leq t\right) P\left(\tau_{1} \leq t\right) .
$$

Notice that $P\left(Z_{t} \leq z \mid \tau_{1}>t\right)=1$, and we apply the law of total probability to $P\left(Z_{t} \leq z \mid \tau_{1} \leq t\right)$. Equation (8) becomes

$$
\begin{gathered}
F(t, z)=P\left(\tau_{1}>t\right)+ \\
+P\left(\tau_{1} \leq t\right) \int_{0}^{t} \int_{0}^{\infty} P\left(Z_{t} \leq z \mid \tau_{1}=u, X_{1}=w\right) f_{X}(w) f_{\tau}\left(u \mid \tau_{1} \leq t\right) d w d u .
\end{gathered}
$$

Now, we split the last double integral as follows,

$$
\begin{gathered}
\int_{0}^{t} \int_{0}^{\infty} P\left(Z_{t} \leq z \mid \tau_{1}=u, X_{1}=w\right) f_{X}(w) f_{\tau}\left(u \mid \tau_{1} \leq t\right) d w d u= \\
\int_{0}^{t} \int_{0}^{z} P\left(Z_{t} \leq z \mid \tau_{1}=u, X_{1}=w\right) f_{X}(w) f_{\tau}\left(u \mid \tau_{1} \leq t\right) d w d u+ \\
\int_{0}^{t} \int_{z}^{\infty} P\left(Z_{t} \leq z \mid \tau_{1}=u, X_{1}=w\right) f_{X}(w) f_{\tau}\left(u \mid \tau_{1} \leq t\right) d w d u
\end{gathered}
$$

Clearly, if $X_{1}>z$ then $P\left(Z_{t} \leq z\right)=0$, so the second part is zero. Now we use the renewal property on the first part to obtain

$$
\begin{gathered}
\int_{0}^{t} \int_{0}^{z} P\left(Z_{t-u} \leq z-w \mid \tau_{1}=u, X_{1}=w\right) f_{X}(w) f_{\tau}\left(u \mid \tau_{1} \leq t\right) d w d u= \\
\int_{0}^{t} \int_{0}^{z} F(t-u, z-w) f_{X}(w) \frac{f_{\tau}(u)}{P\left(\tau_{1} \leq t\right)} d w d u
\end{gathered}
$$

substituting this into (8) yields (7) after a change of variables.

Example. Suppose that $X_{1} \sim \exp (\mu)$ and $\tau_{1} \sim \exp (\lambda)$, i.e. process $Z$ is a Compound Poisson process with exponential jumps. Then we can work out the probabilities in equation (7) to obtain

$$
F(t, z)=e^{-\lambda t}+\int_{0}^{t} \int_{0}^{z} \lambda e^{-\lambda(t-u)} \mu e^{-\mu(z-w)} F(u, w) d w d u .
$$

Solving analytically these integral equations is not an easy task, thus it seems natural appealing to numerical methods. 


\subsection{A numerical example}

We solve numerically equation (9) and we also carry out some simulations in order to have a comparison.

We have taken $\lambda=1$ and $\mu=1$ in equation (9). We made a grid of the region $D=[0,1] \times[0,1]$ by taking a partition of 60 equidistant subintervals on both axis, then we solve iteratively. Table 1 shows the approximated values of $F(t, z)$ at specific nodes.

\begin{tabular}{c|cccc}
\hline \hline$t \backslash z$ & 0.1 & 0.5 & 0.7 & 1 \\
\hline 0.1 & 0.913373 & 0.940521 & 0.950717 & 0.962831 \\
0.5 & 0.635487 & 0.731907 & 0.770276 & 0.817963 \\
0.7 & 0.529976 & 0.643721 & 0.690256 & 0.749334 \\
1 & 0.403539 & 0.52919 & 0.582708 & 0.652746 \\
\hline \hline
\end{tabular}

Table 1: $F(t, z)$.

In order to check the obtained values, additionally, we carry out estimates for $F(t, z)$ on the nodes of Table 1 by means of simulation. That is, we run 100,000 paths for the process $Z_{t}$ for $0 \leq t \leq 1$, then we calculate the proportions $F(t, z)$ for $t=\{.1, .5, .7,1\}$ and $z=\{.1, .5, .7,1\}$. The results are in Table 2 and can be compared with those ones of Table 1.

\section{Discounted random sums}

Now, we are interested in calculating the probability distribution of $Z_{t}^{(\delta)}$, defined in (3). For this model, we use the following notation:

$$
F_{\delta}(t, z)=P\left(Z_{t}^{(\delta)} \leq z\right)
$$

Proposition 2 The following integral equation holds for $F_{\delta}(t, z)$ :

$$
F_{\delta}(t, z)=P\left(\tau_{1}>t\right)+
$$

\begin{tabular}{c|cccc}
\hline \hline$t \backslash z$ & 0.1 & 0.5 & 0.7 & 1 \\
\hline 0.1 & 0.91326 & 0.94076 & 0.95076 & 0.96332 \\
0.5 & 0.6341 & 0.73235 & 0.77147 & 0.82081 \\
0.7 & 0.53114 & 0.64576 & 0.69151 & 0.75113 \\
1 & 0.40435 & 0.52928 & 0.58306 & 0.65277 \\
\hline \hline
\end{tabular}

Table 2: $F(t, z)$. 


$$
\int_{0}^{t} \int_{0}^{z e^{\delta(t-u)}} F_{\delta}(u, w) f_{X_{1}}\left(z e^{\delta(t-u)}-w\right) f_{\tau_{1}}(t-u) d w d u
$$

Proof. Analogously to Proposition 1 we have

$$
F_{\delta}(t, z)=P\left(Z_{t}^{(\delta)} \leq z \mid \tau_{1}>t\right) P\left(\tau_{1}>t\right)+P\left(Z_{t}^{(\delta)} \leq z \mid \tau_{1} \leq t\right) P\left(\tau_{1} \leq t\right) .
$$

Notice that $P\left(Z_{t}^{(\delta)} \leq z \mid \tau_{1}>t\right)=1$. Now, we treat $P\left(Z_{t}^{(\delta)} \leq z \mid \tau_{1} \leq t\right)$ separately,

$$
\begin{aligned}
& P\left(Z_{t}^{(\delta)} \leq z \mid \tau_{1} \leq t\right)=\int_{0}^{t} P\left(Z_{t}^{(\delta)} \leq z \mid \tau_{1}=u\right) f_{\left(\tau_{1} \mid \tau_{1} \leq t\right)}(u) d u \\
& =\int_{0}^{t} \int_{0}^{\infty} P\left(X_{1} e^{-\delta \tau_{1}}+e^{-\delta \tau_{1}} \sum_{i=2}^{N_{t}} X_{i} e^{-\delta T_{i}^{+}} \leq z \mid \tau_{1}=u, X_{1}=w\right) \times \\
& \quad \times f_{X_{1}}(w) f_{\tau_{1}}\left(u \mid \tau_{1} \leq t\right) d w d u
\end{aligned}
$$

where $T_{i}^{+}=\sum_{j=2}^{i} \tau_{j}$. Notice that if $w>z e^{\delta u}$, the probability inside the double integral is naught. This is because once the first claim surpasses the future value of $z$, the total discounted amount will be above $z$ thereafter, because the process is increasing. Therefore, equation (11) resumes into

$$
\int_{0}^{t} \int_{0}^{z e^{\delta u}} P\left(w e^{-\delta u}+e^{-\delta u} \sum_{i=2}^{N_{t}} X_{i} e^{-\delta T_{i}^{+}} \leq z\right) f_{X_{1}}(w) f_{\tau_{1}}\left(u \mid \tau_{1} \leq t\right) d w d u=
$$

(we now apply the renewal property)

$$
\begin{gathered}
\int_{0}^{t} \int_{0}^{z e^{\delta u}} P\left(\sum_{i=1}^{N_{t-u}} X_{i} e^{-\delta T_{i}} \leq z e^{\delta u}-w\right) f_{X_{1}}(w) \frac{f_{\tau_{1}}(u)}{\left.P\left(\tau_{1} \leq t\right)\right)} d w d u= \\
\int_{0}^{t} \int_{0}^{z e^{\delta u}} F_{\delta}\left(t-u, z e^{\delta u}-w\right) f_{X_{1}}(w) \frac{f_{\tau_{1}}(u)}{P\left(\tau_{1} \leq t\right)} d w d u
\end{gathered}
$$

which can be written as in (10) after a change of variable.

Example. Assume that $X_{1} \sim \exp (\mu)$ and $\tau_{1} \sim \exp (\lambda)$, as in the previous example, one can write down a more explicit relation:

$$
F_{\delta}(z, t)=e^{-\lambda t}+\int_{0}^{t} \int_{0}^{z e^{\delta(t-u)}} \lambda e^{-\lambda(t-u)} \mu e^{-\mu\left(z-w e^{\delta(t-u)}\right)} F_{\delta}(w, u) d w d u .
$$

For equation (12), one may use a numerical method to solve it. 


\section{References}

[1] Asmussen, S. (2008) Applied Probability and Queues. Springer, New York.

[2] Atkinson, K. E. (1997) The Numerical Solution of Integral Equations of the Second Kind. Cambridge University Press, Cambridge UK.

[3] Brunner, H.; Kauthen, J.P. (1989) "The numerical solution of twodimensional Volterra integral equations by collocation and iterated collocation", IMA Journal of Numerical Analysis 9(1): 47-59.

[4] Cheney, W. (2001) Analysis for Applied Mathematics. Springer, New York.

[5] Ramsay, C. M. (1994) "On an integral equation for discounted compound-annuity distributions", ASTIN Bulletin 19(2): 191-198.

[6] Rolski, T.; Schmidli, H.; Schmidt, V.; Teugels, J. (1999) Stochastic Processes for Insurance and Finance. John Wiley \& Sons, New York.

[7] Scalas, E. (2006) "Five years of continuous-time random walks in econophysics", in: A. Namatame, T. Kaizouji \& Y. Aruka (Eds.) The Complex Networks of Economic Interactions, Lectures Notes in Economics and Mathematical Systems 567, Springer Verlag, Berlin: 3-16.

[8] Scalas, E.; Gorenflo, R.; Mainardi, F. (2004) "Uncoupled continuoustime random walks: Solution and limiting behavior of the master equation", Physical Review E 69(1): 011107 [8 pages].

[9] Thorin, O.; Wikstad, N. (1973) "Numerical evaluation of the ruin probabilities for a finite period" Astin Bulletin 7: 137-153.

[10] Whitt, W. (2002) Stochastic-Process Limits. An Introduction to Stochastic-Process Limits and Their Applications to Queues. Springer, New York. 
